\title{
Práticas profissionais em situações de violência na atenção domiciliar: revisão integrativa
}

\author{
Professional practices in situations of violence in home care: \\ an integrative review
}

Mariana Almeida Maia (https://orcid.org/0000-0001-6311-2964) ${ }^{1}$

Marina Aparecida Chrispim Silva (https://orcid.org/0000-0002-5769-6024) ${ }^{1}$

Ana Carolina de Oliveira Paiva (https://orcid.org/0000-0001-5729-3658) ${ }^{1}$

Doane Martins da Silva (https://orcid.org/0000-0002-0391-0777) ${ }^{1}$

Marília Alves (https://orcid.org/0000-0002-4695-0787) ${ }^{1}$
${ }^{1}$ Escola de Enfermagem, Universidade Federal de Minas Gerais. Av. Pres. Antônio Carlos 6627, Pampulha. 31270-901 Belo Horizonte MG Brasil. maiamariana.enf@ gmail.com

\begin{abstract}
This article aims to describe the practices of health professionals in situations of violence in the provision of home care. It involved an integrative review of the literature conducted between December 2016 and December 2017 in the LILACS, BDENF and MEDLINE databases. The sample was composed of 15 articles, organized and characterized by author, publication journal, country, year, title, method, main idea, category and level of evidence. The violent situations found most often were abuse of elderly and children and domestic violence towards women and children. Different practices were adopted during violent situations against patients, like interventions, notifications, orientation, and professional qualification. Practices of health professionals in home care focuses on actions of patient care,seek to minimize the effects of violence.
\end{abstract}

Key words Professional practice, Violence, Home care
Resumo O objetivo deste artigo é descrever as práticas de profissionais de saúde em situações de violência nos cuidados da Atenção domiciliar. Trata-se de uma revisão integrativa da literatura realizada entre dezembro de 2016 e dezembro de 2017 nas bases de dados LILACS, BDENF e MEDLINE. A amostra foi composta por 15 artigos, organizados e caracterizados de acordo com autor, periódico de publicação, país, ano, título, método, ideia central, categoria e nivel de evidência. As situações de violência mais encontradas foram abuso em idosos e crianças e violência doméstica em mulheres e crianças. Práticas distintas foram adotadas nas situações de violência contra os pacientes, como intervenções, notificações, orientação, $e$ capacitação profissional. As práticas dos profissionais na atenção domiciliar focalizam ações de cuidado dos pacientes, buscando minimizar os efeitos da violência.

Palavras-chave Prática profissional, Violência, Assistência domiciliar 


\section{Introdução}

A violência é um problema de saúde pública e os profissionais de saúde necessitam estar capacitados para lidar com as situações em seu trabalho ${ }^{1}$. $\mathrm{Na}$ Atenção domiciliar (AD) os profissionais se deparam com situações de violência em seu caráter particular, ou seja, na residência do paciente, geralmente grave, cuja família ou cuidador assume os cuidados planejados pelas equipes. As situações de violência contra diferentes vítimas, de diversas faixas etárias e quadros clínicos, podem não ser percebidas pelos profissionais ou as intervenções implicam na atuação de várias áreas que, se não acompanhadas, podem resultar em prejuízos para as vítimas. Assim, práticas profissionais em situações de violência na $\mathrm{AD}$ constituem um problema de alta complexidade.

Dentre as práticas profissionais na $\mathrm{AD}$, além de cuidar dos pacientes no domicílio, os profissionais ajudam a fazer a gestão do cuidado, realizar a articulação dos pontos de atenção de modo a ampliar a resolutividade e a integralidade do cuidado. O Ministério da Saúde, visando estratégias de prevenção, disponibiliza cursos de capacitação profissional para identificar e lidar com situações na $\mathrm{AD}$. A equipe por meio do atendimento multidisciplinar, busca uma abordagem terapêutica e estabelece as medidas gerais e legais cabíveis a cada caso ${ }^{2}$.

Violência pode ser definida como o uso intencional da força física contra si próprio, contra outra pessoa ou contra um grupo ou comunidade, que possa resultar em lesão, morte, dano psicológico, deficiência de desenvolvimento ou privação. O conceito é muito debatido e polissêmico pois suas definições diferem nas diversas correntes de pensamento, como as biológicas, históricas, culturais, econômicas, psicológicas e outras ${ }^{3}$. Além de possuir altos custos para a área de saúde com internações, cirurgias, materiais e tratamentos que precisarão ser feitos durante toda a vida do usuário ${ }^{4}$.

É uma questão de grande amplitude cujo enfrentamento envolve profissionais de diferentes campos de atuação, requerendo efetiva mobilização de diversos setores do governo e da sociedade civil. Tal mobilização visa fortalecer e potencializar as práticas nos serviços na perspectiva de uma nova atitude, compromisso e colaboração em relação ao problema ${ }^{5}$. Há vários tipos de violência que podem ser definidos pelo tipo de ação praticada no indivíduo como a violência física, moral, sexual entre outras, além de atingir crianças, adolescentes, mulheres e idosos em diferentes espaços sociais.
Entre os espaços de cuidados à saúde encontra-se a AD, uma modalidade de atenção da Rede de Atenção à Saúde, caracterizada por um conjunto de ações de prevenção, tratamento, reabilitação, paliação e promoção à saúde, prestadas em domicílio, visando a continuidade dos cuidados. Evita hospitalizações desnecessárias e pode apoiar equipes da atenção básica em favor do aumento da eficiência da assistência ${ }^{5,6}$. Apresenta o lar como um espaço de cuidado, onde o tempo de contato entre os profissionais de saúde e paciente pode proporcionar condições para detectar maus tratos e poder intervir.

$\mathrm{Na}$ área de saúde os profissionais devem estar atentos para identificar casos de violência e maus-tratos para efetivar ações de prevenção, promoção e acolhimento. É imprescindível que os conceitos de violência sejam difundidos entre os integrantes da equipe, assim como para a comunidade ${ }^{5}$. Um único incidente de maus tratos pode desencadear uma espiral crescente que leva à perda de independência, doença grave ou até a morte. A violência praticada contra idosos, crianças ou mulheres tem implicações na saúde e bem-estar porque as vítimas têm menos opções para evitar situações abusivas por causa da idade, condição de saúde ou recursos limitados, tornando estes grupos mais vulneráveis e menos capazes de se recuperar da vitimização ${ }^{6}$.

Por outro lado, "a violência contra adolescentes nas comunidades e nas ruas é um fenômeno tipicamente urbano e fortemente determinado pelas desigualdades sociais e econômicas nesses espaços", como assassinatos, acidentes de trânsito e exploração sexual ${ }^{7}$. Nas famílias, a violência física contra adolescentes é considerada um mal silencioso, haja vista que as vítimas podem não conhecer ou ter coragem de buscar maneiras de resolver o problema ${ }^{8}$. Neste estudo a violência contra adolescentes não será focalizada por não terem sido encontrados artigos sobre adolescentes em $\mathrm{AD}$, mas focalizando grupos de idosos, crianças e mulheres em AD.

Neste sentido, a questão norteadora deste estudo foi "Como são as práticas dos profissionais de saúde da $\mathrm{AD}$ em situações de violência?" e tem como objetivo descrever as práticas de profissionais de saúde em situações de violência nos cuidados da Atenção domiciliar.

\section{Método}

A revisão integrativa da literatura é um método de pesquisa que permite a síntese de múltiplos estudos publicados, incluindo o aprofundamen- 
to de pesquisas que sustentam a tomada de decisão e a melhoria da prática clínica. Possibilita a definição de conceitos, revisão das teorias, avaliação de evidências e análise de questões metodológicas sobre determinado tema ${ }^{9}$.

Este estudo se configura como uma revisão integrativa da literatura, cuja questão norteadora foi estruturada por meio da estratégia $\mathrm{PICO}^{10}$ : (P) Paciente ou Problema - violência na atenção domiciliar; (I) Intervenção - práticas dos profissionais de saúde; (C) Controle ou Comparação - não se aplica a este estudo; (O) Desfecho - estratégias desenvolvidas para enfrentamento da violência na Atenção domiciliar. Para sua operacionalização, foram consideradas as seguintes etapas: elaboração da questão norteadora; estabelecimento dos critérios de inclusão e exclusão de estudos; definição das informações a serem extraídas dos estudos selecionados; avaliação dos estudos incluídos na revisão; interpretação dos resultados e apresentação da revisão/síntese do conhecimento $^{11}$.

A coleta foi realizada em publicações do período de dezembro de 2016 a dezembro de 2017 e foram utilizadas as bases de dados: Medical Literature Analysis and Retrieval System Online (MEDLINE), consultada por meio do PubMed e a Base de Dados Específica da Enfermagem (BDENF) e Literatura Latino-Americana e do Caribe em Ciências da Saúde (LILACS), consultados pela Biblioteca Virtual em Saúde (BVS), minimizando possíveis vieses.

Foram incluídos os artigos publicados em inglês, espanhol e português, texto completo e que apresentavam títulos e resumos que abordavam as práticas profissionais em situações de violência na $\mathrm{AD}$. Foram excluídos documentos, dissertações, teses, manuais oficiais e relatórios técnicos que não abordavam a violência identificada na atenção domiciliar. Estudos cujos pacientes fossem tratados fora de casa, como asilos e/ou creches (out-home care) foram excluídos.

Por violência se tratar de um fenômeno complexo e difícil de conceituar, adotou-se descritores que se aproximassem com o termo, além de descritores sobre AD. Assim, na estratégia de busca na PubMed e BVS utilizou-se os termos descritos no Quadro 1.

$\mathrm{Na}$ busca inicial, encontrou-se um total de 488 artigos. Pela leitura dos títulos e resumos, foi possível excluir 22 artigos por duplicidade nas diferentes bases e 365 artigos que não atendiam aos critérios de inclusão. Nesta etapa de elegibilidade encontrou-se 132 artigos que foram analisados e 88 artigos foram excluídos por não corresponder à questão norteadora ou não serem localizados na íntegra. Assim, dos 44 artigos lidos na íntegra, 29 foram excluídos por abordarem sobre out-home care e não atenderem ao objetivo da revisão. Desta forma, 15 artigos responderam à questão norteadora e constituíram a amostra desta revisão, conforme a Figura 1.

Para a seleção das publicações, a leitura dos artigos na íntegra foi avaliada por dois pesquisadores que possuem expertise na área, seguindo os critérios de inclusão e exclusão e norteados pela pergunta de pesquisa. Cada pesquisador registrou sua avaliação e justificativa de inclusão ou exclusão do artigo em um banco de dados, detalhado por título, revista, base de dados, autores, resumo, resposta à questão e justificativa de inclusão ou exclusão. Ainda nesta etapa, os resultados foram discutidos e as discordâncias solucionadas por consenso entre os pesquisadores.

Na etapa de análise dos artigos, optou-se por discuti-los pelas dimensões de práticas do cuidado em idosos, crianças e mulheres. Esse critério temático foi utilizado por observar que as situações de violências nas diferentes etapas de vida demandam ações de conduta distintas pelos profissionais de saúde.

Avaliou-se a qualidade dos artigos com base na classificação do nível de evidência da Agency for Healthcare Research and Quality (AHRQ). A qualidade das evidências é classificada em seis níveis, sendo: I - Evidências resultantes da meta -análise de múltiplos estudos clínicos controlados e randomizados; II - Evidências obtidas em estudos individuais com delineamento experimental; III - Evidências de estudos quase-experimentais; IV - Evidências de estudos descritivos ou com abordagem qualitativa; V - Evidências provenientes de relatos de caso ou de experiência e VI - Evidências baseadas em opiniões de especialistas $^{12}$.

Por tratar-se de revisão integrativa da literatura, não houve necessidade de análise por Comitê de Ética em Pesquisa (CEP). Foi mantida a autenticidade das ideias, conceito e definições dos autores dos artigos.

\section{Resultados e discussão}

Os artigos foram lidos na íntegra e agrupados por similaridade de conteúdo, sob a forma de categorias empíricas, sendo construídas três categorias para análise, assim especificadas: Práticas profissionais em situações de violência identificadas contra idosos (I), práticas relacionadas a criança 
Quadro 1. Sintaxe dos descritores utilizados para a estratégia de busca. Belo Horizonte, 2018.

("Violência Doméstica" OR "Domestic Violence" OR "Violencia Doméstica" OR "MausTratos Infantis" OR "Child Abuse" OR "Maltrato a los Niños" OR "Maus-Tratos ao Idoso" OR "Elder Abuse" OR "Maltrato al Anciano" OR "Maus-Tratos Conjugais" OR "Spouse Abuse" OR "Maltrato Conyugal" OR"Delitos Sexuais" OR "Sex Offenses" OR "Delitos Sexuales" OR "Abuso Sexual na Infância" OR "Child Abuse, Sexual" OR "Child Sexual Abuse" OR "Abuso Sexual Infantil" OR Estupro OR Rape OR Violación OR "Violência contra a Mulher" OR "Violence Against Women" OR "Violencia contra la Mujer") AND ("Assistência Domiciliar" OR "Home Nursing" OR "Atención Domiciliaria de Salud" OR "Serviços de Assistência Domiciliar" OR "Home Care Services" OR "Servicios de Atención de Salud a Domicilio" OR "Cuidados Intermitentes" OR "Respite Care" OR "atenção domiciliar" OR "home care")

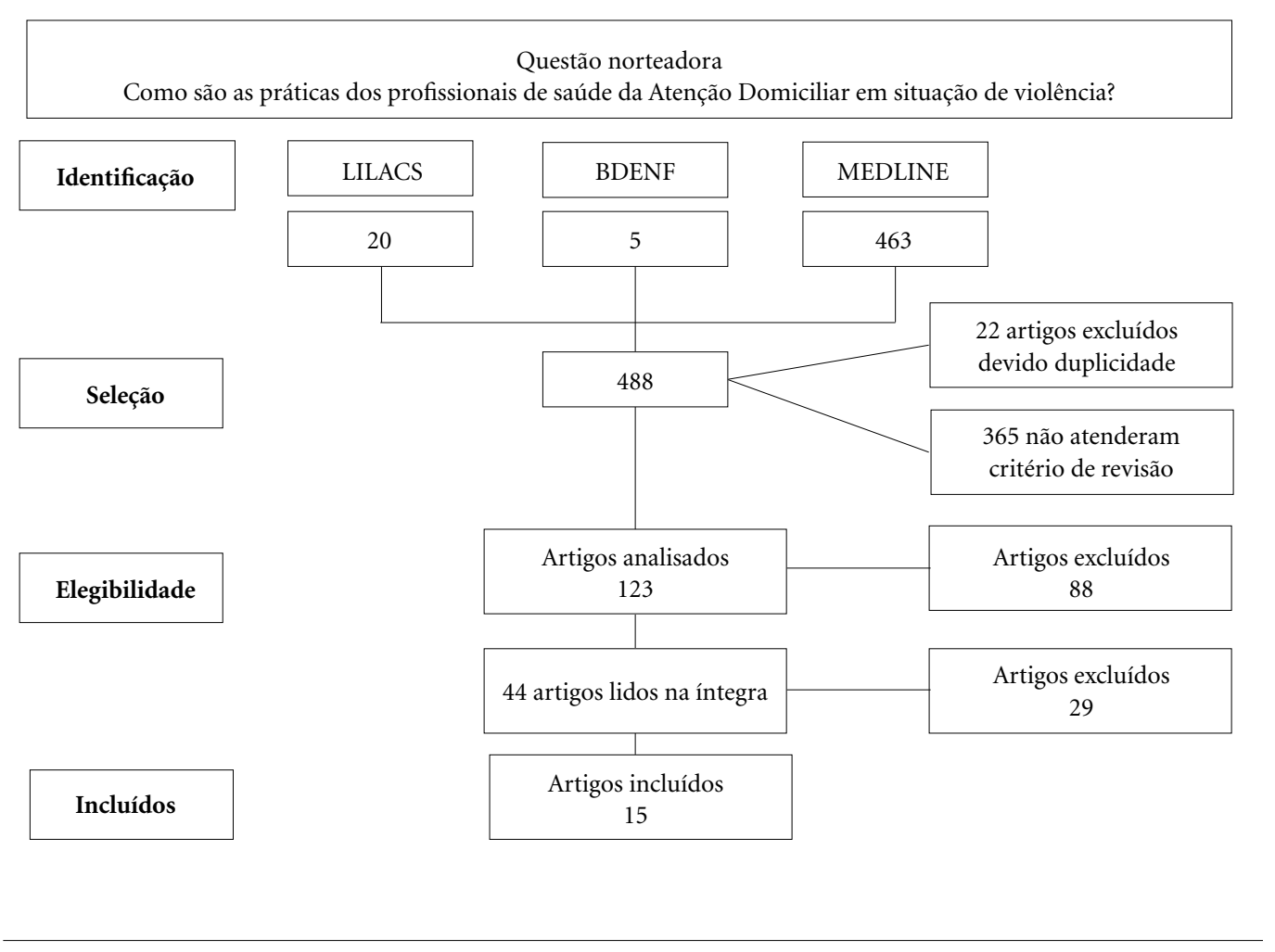

Figura 1. Fluxograma de seleção de artigos para revisão integrativa. Belo Horizonte, 2018.

Fonte: Fluxograma elaborado pelas autoras.

(II) e práticas relacionadas a mulher (III). Desta forma, apresenta-se o Quadro 2 com a descrição dos artigos, classificação quanto ao nível de evidência e categoria temática abordada.

Foram analisados 15 estudos, entre os quais a abordagem qualitativa foi predominante $(\mathrm{n}=$ $7)$, seguidos da abordagem quantitativa $(n=6)$ e revisões $(n=2)$. Os estudos foram realizados nos Estados Unidos da América $(n=11 / 73.3 \%)$,
Austrália $(\mathrm{n}=2 / 13.3 \%)$, Canadá $(\mathrm{n}=1 / 6.7 \%)$ e Israel $(n=1 / 6.7 \%)$. Entre os tipos de violência descritos nos artigos incorporados à revisão destacam-se abordagens sobre abuso, maus tratos e negligência contra crianças e idosos e a violência intrafamiliar contra crianças e mulheres. A maioria dos estudos apresentou nível de evidência IV (40\%) que são estudos descritivos ou com abordagem qualitativa. 
Quadro 2. Descrição dos artigos segundo autor, periódico de publicação, ano, país, título, ideia central e nível de evidência (NE). Belo Horizonte, 2018.

\begin{tabular}{|c|c|c|c|}
\hline Autor. Periódico. Ano. País & Título & Ideia Central & NE \\
\hline $\begin{array}{l}\text { Pickeringa CEZ, et al. } \\
\text { International Journal of } \\
\text { Nursing Studies. } 2017 . \\
\text { EUA }^{13}\end{array}$ & $\begin{array}{l}\text { Identifying elder abuse \& neglect } \\
\text { among family caregiving dyads: } \\
\text { A cross sectional study of } \\
\text { psychometric properties of the } \\
\text { QualCare scale }\end{array}$ & $\begin{array}{l}\text { Apresenta a utilização do instrumento } \\
\text { de escala "QualCare Scale" na } \\
\text { identificação de abuso de idosos em } \\
\text { domicílio pelos profissionais de saúde }\end{array}$ & III \\
\hline $\begin{array}{l}\text { Couture M, et al. Journal } \\
\text { of Interpersonal Violence. } \\
\text { 2016. Canadá }{ }^{14}\end{array}$ & $\begin{array}{l}\text { Implementing a Systematic } \\
\text { Screening Procedure for Older } \\
\text { Adult Mistreatment Within } \\
\text { individual Clinical Supervision: } \\
\text { Is It Feasible? }\end{array}$ & $\begin{array}{l}\text { Apresenta o processo de } \\
\text { implementação de um novo } \\
\text { procedimento de triagem sistemática } \\
\text { para maus tratos de idosos. }\end{array}$ & III \\
\hline $\begin{array}{l}\text { Sheehan OC. et al. Journal } \\
\text { of The American Geriatrics } \\
\text { Society. 2016. EUA }{ }^{15}\end{array}$ & $\begin{array}{l}\text { Development of Quality } \\
\text { Indicators to Address Abuse and } \\
\text { Neglect in Home-Based Primary } \\
\text { Care and Palliative Care }\end{array}$ & $\begin{array}{l}\text { Apresenta estudos que utilizaram o } \\
\text { padrão de qualidade QI na abordagem } \\
\text { de abuso e negligência de idosos } \\
\text { atendidos em cuidados primários } \\
\text { domiciliares e paliativos }\end{array}$ & $\mathrm{V}$ \\
\hline $\begin{array}{l}\text { Johnson YO, Home } \\
\text { Healthcare Now. } 2015 . \\
\text { EUA }^{16}\end{array}$ & $\begin{array}{l}\text { Home Care Nurses' Experiences } \\
\text { With and Perceptions of elder } \\
\text { self-neglect }\end{array}$ & $\begin{array}{l}\text { Apresenta os determinantes de } \\
\text { percepção das enfermeiras domiciliares } \\
\text { sobre a autonegligência de idosos, } \\
\text { suas experiências e as etapas que os } \\
\text { enfermeiros tomam quando se suspeita } \\
\text { de autonegligência. }\end{array}$ & IV \\
\hline $\begin{array}{l}\text { Vognar L, Gibbs LM. } \\
\text { Clinics in Geriatric } \\
\text { Medicine. 2014.EUA } \\
\end{array}$ & Care of the Victim & $\begin{array}{l}\text { Apresenta o treinamento para } \\
\text { profissionais de saúde na detecção e } \\
\text { conduta de abuso de idosos }\end{array}$ & IV \\
\hline $\begin{array}{l}\text { Powers JS. Clinics in } \\
\text { Geriatric Medicine. } 2014 \\
\text { EUA }^{17}\end{array}$ & $\begin{array}{l}\text { Common Presentations of Elder } \\
\text { Abuse in Health Care Settings }\end{array}$ & $\begin{array}{l}\text { Apresenta práticas das instituições na } \\
\text { identificação de abuso de idosos }\end{array}$ & IV \\
\hline $\begin{array}{l}\text { Sharps P, et al. Pediatrics. } \\
\text { 2014. EUA }{ }^{18}\end{array}$ & $\begin{array}{l}\text { Engaging and Retaining Abused } \\
\text { Women in Perinatal Home } \\
\text { Visitation Programs }\end{array}$ & $\begin{array}{l}\text { Apresenta o Programa de Visitas } \\
\text { domiciliares para promoção e } \\
\text { prevenção da violência doméstica } \\
\text { contra as mulheres grávidas. }\end{array}$ & II \\
\hline $\begin{array}{l}\text { Ayalon L. Journals of } \\
\text { Gerontology, Series B: } \\
\text { Psychological Sciences and } \\
\text { Social Sciences. 2014. Israel }{ }^{19}\end{array}$ & $\begin{array}{l}\text { Reports of Elder Neglect by Older } \\
\text { Adults, Their Family Caregivers, } \\
\text { and Their Home Care Workers: A } \\
\text { Test of Measurement Invariance } \\
\end{array}$ & $\begin{array}{l}\text { Apresenta a Escala } 7 \text {-item para } \\
\text { identificação e prevenção da violência } \\
\text { em idosos }\end{array}$ & II \\
\hline $\begin{array}{l}\text { Cairns J, Vreugdenhil A. } \\
\text { Australasian Journal on } \\
\text { Ageing. 2014. Austrália }{ }^{20} \\
\end{array}$ & $\begin{array}{l}\text { Working at the frontline in cases } \\
\text { of elder abuse: 'It keeps me awake } \\
\text { at night' }\end{array}$ & $\begin{array}{l}\text { Apresenta as práticas de saúde dos } \\
\text { profissionais da Tasmânia frente ao } \\
\text { bem-estar de idosos que sofrem abuso }\end{array}$ & IV \\
\hline $\begin{array}{l}\text { Friedman B, et al. Journal } \\
\text { of Elder Abuse \& Neglect. } \\
\text { 2015. EUA }{ }^{21}\end{array}$ & $\begin{array}{l}\text { Longitudinal Prevalence and } \\
\text { Correlates of Elder Mistreatment } \\
\text { Among Older Adults Receiving } \\
\text { Home Visiting Nursing }\end{array}$ & $\begin{array}{l}\text { Apresenta a prevalência de maus tratos } \\
\text { em idosos, os subtipos de maus tratos e } \\
\text { fatores associados }\end{array}$ & I \\
\hline $\begin{array}{l}\text { Alonso-Marsden S, et al. } \\
\text { Child Abuse \& Neglect. } \\
\text { 2013. EUA }{ }^{22}\end{array}$ & $\begin{array}{l}\text { Family risk as a predictor of } \\
\text { initial engagement and follow- } \\
\text { through in a universal nurse } \\
\text { home visiting program to prevent } \\
\text { child maltreatment }\end{array}$ & $\begin{array}{l}\text { Apresenta avaliação de um programa } \\
\text { de visita domiciliar como estratégia de } \\
\text { identificação e prevenção da violência } \\
\text { doméstica em crianças }\end{array}$ & III \\
\hline $\begin{array}{l}\text { Davidov DM, et al. Public } \\
\text { Health Nursing. } 2012 . \\
\text { EUA }^{23}\end{array}$ & $\begin{array}{l}\text { Nurse Home Visitors' } \\
\text { Perspectives of Mandatory } \\
\text { Reporting of Children's Exposure } \\
\text { to Intimate Partner Violence to } \\
\text { Child Protection Agencies }\end{array}$ & $\begin{array}{l}\text { Apresenta como os enfermeiros são } \\
\text { treinados para identificar crianças que } \\
\text { possam sofrer abuso ou negligência } \\
\text { infantil e os procedimentos de relatório } \\
\text { para casos suspeitos de maus tratos } \\
\text { infantis. }\end{array}$ & III \\
\hline
\end{tabular}


Quadro 2. Descrição dos artigos segundo autor, periódico de publicação, ano, país, título, ideia central e nível de evidência (NE). Belo Horizonte, 2018.

\begin{tabular}{|l|l|l|c|}
\hline Autor. Periódico. Ano. País & \multicolumn{1}{|c|}{ Título } & \multicolumn{1}{|c|}{ Ideia Central } & NE \\
\hline $\begin{array}{l}\text { Davidov DM, et al. } \\
\text { Violence Against Women. } \\
\text { 2012. EUA }{ }^{24}\end{array}$ & $\begin{array}{l}\text { Mandatory Reporting in the } \\
\text { Context of Home Visitation } \\
\text { Programs: Intimate Partner } \\
\text { Violence and Childre7n's } \\
\text { Exposure to Intimate Partner } \\
\text { Violence }\end{array}$ & $\begin{array}{l}\text { Apresenta os problemas relacionados } \\
\text { de preenchimento dos relatórios } \\
\text { obrigatórios de identificação de abuso } \\
\text { de crianças na visita domiciliar. }\end{array}$ & IV \\
\hline $\begin{array}{l}\text { Ammerman RT, et al. } \\
\text { Behavior Modification. } \\
\text { 2012. EUA }{ }^{25}\end{array}$ & $\begin{array}{l}\text { Predictors of Treatment Response } \\
\text { in Depressed Mothers Receiving } \\
\text { In-Home Cognitive-Behavioral } \\
\text { Therapy and Concurrent Home } \\
\text { Visiting }\end{array}$ & $\begin{array}{l}\text { Apresenta a visita domiciliar como } \\
\text { stratégia de prevenção da violência } \\
\text { doméstica, estudo com mulheres }\end{array}$ & IV \\
\hline $\begin{array}{l}\text { Segal L, Opie R, Dalziel K. } \\
\text { The Milbank Quarterly } \\
\text { 2012. Austrália }{ }^{26}\end{array}$ & $\begin{array}{l}\text { Theory! The Missing Link in } \\
\text { Understanding the Performance } \\
\text { of Neonate/Infant Home-Visiting } \\
\text { Programs to Prevent Child } \\
\text { Maltreatment: Systematic Review }\end{array}$ & $\begin{array}{l}\text { Apresenta a visita domiciliar como } \\
\text { estratégia para redução de abuso } \\
\text { infantil }\end{array}$ & V \\
\hline
\end{tabular}

Práticas profissionais em situações de violência identificadas contra idosos

Os estudos foram realizados em países desenvolvidos, com práticas de profissionais e cuidadores domiciliares vinculados aos serviços de saúde e de assistência social. Segundo a maioria dos estudos ${ }^{13-17}$, alguns idosos não são capazes de realizar atividades básicas de vida diária sozinhos, como comer ou ir ao banheiro, ou seja, são indivíduos dependentes de cuidado. Além disso, o abuso de idosos é um problema que cresceu ao longo de décadas no mundo e que continua crescendo à medida que a população mundial envelhece de 35 milhões para uma previsão de 72 milhões até $2030^{27}$.

Os idosos em situação de violência possuem dificuldades para tarefas básicas, hematomas sem explicação, sujos, desidratados e famintos. O idoso dependente de cuidados altera a dinâmica familiar e pode ser acometido por maus tratos pelo despreparo da família em assumir seus cuidados. Essa situação pode levar cuidadores familiares a situações de estresse e negligência no atendimento às necessidades, sendo possível chegar a abusos físicos, financeiros, psicológicos e de maus-tratos.

Um estudo buscou identificar práticas que os profissionais adotavam diante de casos de autonegligência de idosos, relacionados à dificuldade em seus cuidados como necessidades básicas, higiene pessoal inadequada, acúmulos de objetos, má alimentação e não procurar auxílio médico quando necessário. Os sentimentos de choque da realidade, como "horrível", "triste" e "frustrante" devido as condições como os pacientes eram encontrados em casa, foram descritos pelos profissionais além de tristeza ou culpa pela incapacidade de solucionar os problemas encontrados. As práticas desenvolvidas envolvem o pensamento crítico sobre a situação do idoso em suas necessidades percebidas, agir com ética e carinho, educação em saúde, atenção à saúde mental dos idosos e na sua capacidade de tomar decisões. Os profissionais por inexperiência profissional ou ausência de orientações para o cuidado na formação sentem-se incapacitados para intervir de forma eficaz diante das situações de autonegligência de idosos ${ }^{16}$.

No estudo realizado em Israel, os resultados apontam o uso da escala de negligência como prática na identificação e avaliação de idosos negligenciados em uma unidade cuidadora. Além disso, os profissionais de cuidados domiciliários podem abster-se de relatar a negligência e/ou não assumir o cuidado como sua responsabilidade porque o relato de uma suspeita de negligência pode colocá-los em risco de perder o emprego ${ }^{19}$.

Um estudo canadense aponta como prática um procedimento de triagem sistemática de sessões de supervisão clínica individual para apoiar assistentes sociais a melhorar a assistência aos idosos na detecção de maus-tratos. Contudo, esta 
atividade compete com as atividades administrativas, nas quais o procedimento poderia ir além da triagem e que ainda há uma dificuldade de registro das informações de maus tratos a idosos. $\mathrm{O}$ processo sensibiliza os assistentes sociais quanto aos fatores de risco para situações de violência ao idoso, o que permite refletir sobre as ações e discutir os casos em grupos ${ }^{14}$. Neste contexto, a identificação de maus tratos a idosos e intervenções sobre os fatores de risco reduzem a ocorrência das situações de violência.

Estudo realizado nos Estados Unidos apresentou como prática o planejamento individualizado de cuidados centrados no paciente, com o objetivo de propor um ambiente terapêutico de cuidados, comunicação aprimorada da equipe e mudança de protocolo de comunicação. Os resultados apontaram estas mudanças como estratégias úteis para enfrentar o problema de abuso contra idosos ${ }^{17}$.

Um dos estudos selecionados teve como objetivo apontar indicadores de qualidade sobre abuso e negligência em cuidados domiciliares. Foram identificadas três principais práticas profissionais: desenvolvimento de diretrizes que indicam como abordar os abusos, provisão de educação e treinamento para equipe de saúde e envolvimento de especialistas interdisciplinares e equipes na abordagem de abusos. Foram apontadas diferenças nos quesitos de notificação obrigatória, falta de médicos conscientizados com as políticas e dúvidas sobre o que relatar e para quem, o que contribui para subnotificação do abuso de idosos ${ }^{15}$.

Ainda com relação aos idosos, outras práticas utilizadas diante de situação de violência são as avaliações médicas para questões agudas e de capacidade, avaliação para tutela, educação dos pacientes e familiares e resolução de problemas. Os profissionais de saúde, quando detectam necessidade de intervenção legal, acionam outros serviços para abordar as questões legais das vítimas que sofreram abuso, negligência ou exploração e precisam de proteção e tutela ${ }^{6}$.

Para os profissionais de saúde trabalhar com casos de abuso de idosos é desafiador, frustrante e o trabalho é difícil e às vezes perigoso. No cotidiano, enfrentam desafios em seu trabalho com quem sofre abuso como intimidação e ameaça (dos membros da família); dilemas práticos (equilíbrio no dever de cuidar com a autodeterminação do paciente); e apoio organizacional. Neste contexto, as demandas dos pacientes necessitam de políticas organizacionais contra abuso de idosos, gerenciamento de apoio e serviços de apoio à violência familiar e etária ${ }^{19}$.
Um estudo abordou a visita domiciliar como uma estratégia assistencial que permite ao profissional de saúde e paciente desenvolver vínculos para que o paciente confie no profissional o suficiente para revelar que está sendo maltratado. $\mathrm{O}$ vínculo é desenvolvido no tempo que o profissional de saúde passa com o paciente e estabelece confiança entre ambos, resultando em uma assistência com diálogo. Um dos achados do estudo aponta que 10,5 visitas mensais, com duração média de uma hora por visita, podem facilitar o discernimento de abuso contra os idosos, o que torna a negligência percebida como forma do profissional perceber maus tratos ${ }^{20}$.

A sensibilização sobre fatores de risco de abuso de idosos e a variedade de intervenções em diferentes contextos ajudam a promover a detecção, tratamento e prevenção dos abusos. Para a manutenção do ambiente doméstico a vítima pode ser levada para assistência domiciliar temporária ou outros (lar adotivo, lar de idosos ou hospital) por vários dias a semanas o que pode permitir um período de descanso para os cuidadores familiares. A assistência domiciliar temporária pode incluir: refeições, administração de medicamentos transporte, lembretes de saúde e cuidado pessoal ${ }^{17}$.

Alguns instrumentos podem ser aplicados para ajudar na identificação dos abusos em idosos como a Qualcare Scale, projetada para fornecer uma medida quantificável da qualidade da assistência prestada pelos cuidadores familiares. A escala possui 52 itens e está organizada em seis dimensões incluindo cuidados ambientais, cuidados físicos, manutenção de cuidados médicos, cuidados psicológicos, violações dos direitos humanos e financeiro. O instrumento de rastreamento é promissor para detectar os tipos de cuidados aos idosos e facilitar que os profissionais de saúde que prestam atendimento domiciliar, com o tempo, desenvolvam vínculos com o paciente e suas famílias. Fazer várias visitas durante um período torna a Qualcare Scale uma ferramenta viável para ser implementada na prática da $\mathrm{AD}^{13}$.

A identificação de abuso no domicílio requer um alto nível de suspeita e que os incidentes na instalação de cuidados sejam diretamente observáveis e rapidamente descobertos. O ambiente doméstico revela pistas sobre os abusos cometidos que são mais difíceis de serem percebidas em visitas ao consultório. Os pacientes idosos que se apresentam no consultório podem não ser capazes de revelar todas as preocupações, especialmente se acompanhados pelo cuidador. Sinais reveladores de medo, silêncio ou incapacidade 
de conversar com o paciente idoso sozinho são sinais de alerta de possíveis abusos para o profissional. $\mathrm{O}$ abuso pode assumir muitas formas e muitas vezes não é diretamente observável.

\section{Práticas profissionais em situações de violência identificadas contra crianças}

Os artigos apresentam consenso quanto a importância da visita domiciliar e da notificação como uma prática adotada tanto para identificar casos de violência quanto para a prevenção de violência contra crianças. Além disso, abordam a necessidade da ação de profissionais de saúde específicos para enfrentar a violência relacionada às crianças s2-24,26. $^{2}$.

As visitas domiciliares, consultas médicas e a coleta de informações de terceiros são os principais momentos de identificação da violência doméstica contra a criança. O domicílio se torna um ambiente suscetível à ocorrência de violência infanto-juvenil uma vez que mais da metade $(52,3 \%)$ ocorreu no domicílio e foi causada por um membro da família $(63,2 \%)^{28}$.

Um estudo selecionado teve como objetivo compreender se as visitas domiciliares auxiliam na deteç̧ão de maus tratos contra crianças. As evidências apontaram que as visitas domiciliares realizadas no período de pré-natal e durante a infância permitem aos profissionais perceber se o ambiente é seguro, o que a torna um artifício na prevenção e redução de índices de maus tratos infantis ${ }^{26}$. Assim, torna-se possível identificar casos de violência no domicílio e utilizar a denúncia dos mesmos como uma prática adotada pelos enfermeiros no caso de violência entre parceiros em que os filhos são afetados ${ }^{23}$. No Brasil, a atuação de Agentes Comunitários de Saúde no domicílio favorece a aproximação e o vínculo entre comunidade e equipe de saúde e, por ser um elo de interlocução, seu papel é estratégico, pois disponibiliza informações sobre problemas contextuais das famílias que estão expostas à violência ${ }^{29}$.

Estudo realizado nos Estados Unidos apontou que de uma amostra de 532 profissionais, aproximadamente $55 \%$ dos enfermeiros deste estudo, que realizavam visitas domiciliares, relataram que devem denunciar a exposição da criança à violência entre parceiros. A maioria dos profissionais relataram que a denúncia pode proteger a criança (92\%) e 56\% indicaram que a denúncia pode afetar a relação profissional-paciente de forma negativa ${ }^{24}$. No contexto brasileiro, a visita domiciliar como ação educativa, envolve ações direcionadas aos pais nas quais são informados em relação ao direito da criança de crescer sem violência e os efeitos da mesma na saúde das crianças, além de utilizar o momento para esclarecer os deveres do responsável em relação à segurança e o bem-estar das crianças ${ }^{30}$.

\section{Práticas profissionais em situações de violência identificadas contra mulheres}

Dos 15 artigos selecionados, dois apontam que os profissionais de saúde utilizam a notificação obrigatória e programas de visitas domiciliares como práticas para lidar com a violência contra mulheres ${ }^{22-26}$. Além disso, outras estratégias vêm sendo adotadas pelos profissionais nos serviços e nas comunidades, entre elas grupos de mulheres, orientações e acolhimento das vítimas, visando melhorar as condições, embora não haja um padrão nas ações dos profissionais frente a situações de violência contra mulheres.

Os programas de visitas domiciliares têm surgido como uma intervenção para melhorar a oferta de serviços de atenção à saúde, bem-estar e prevenção de situações de violência que afetam as puérperas e famílias com recém-nascidos e crianças, particularmente entre população em desvantagens social e econômica em situações de risco relacionado à violência ${ }^{23-25}$.

O programa DOVE (Domestic Violence Enhanced Home Visitation Program) nos Estados Unidos é uma intervenção estruturada em ambientes urbanos e rurais que tem como objetivo identificar e manter mulheres grávidas abusadas em programas de visitação domiciliar perinatais. O estudo apresenta uma visão do programa que demonstra que com treinamento adequado, os profissionais de saúde se sentem confiantes em perceber quando há abusos ou maus tratos no domicílio, e, desta forma, diminuindo a violência entre parceiros íntimos e sua exposição às crianças ${ }^{18}$.

Além disso, as visitas domiciliares podem ser consideradas práticas informativas, pois trazem à luz alguns riscos e benefícios relacionados ao cumprimento da notificação obrigatória e permitem o desvelamento da violência vivida ${ }^{23,31}$.

Um estudo nos Estados Unidos apontou consenso entre os profissionais de saúde em relação à notificação de situações de violência como prática. Revelaram que ao encontrarem situações de abuso e violência entre parceiros íntimos, não há obrigatoriedade dos profissionais em fazer esta notificação à polícia ou aos serviços de proteção para adultos. Em contrapartida, em relação à notificação obrigatória relacionada à exposição da criança a violência entre parceiros, os profissio- 
nais de saúde informaram que sempre realizam a notificação ${ }^{24}$.

Em estudo no contexto brasileiro, profissionais de equipe de Saúde da Família definiram sua atuação frente à violência contra mulheres como uma impotência paralisante reflexo da falta de organização do serviço para este fim. Após uma oficina com situações de violência para preparar os profissionais foi evidenciado que a violência sexual contra a mulher envolve questões nas dimensões singular, particular e estrutural da realidade objetiva que merecem ser refletidas ${ }^{32}$. Para refletir sobre a violência sexual contra crianças e adolescentes, focalizando a atuação do enfermeiro à luz da sua prática profissional e da legislação brasileira, um estudo apontou que enfermeiras expressaram medo, insegurança, desconhecimento e incapacidade no atendimento às mulheres em situação de violência ${ }^{30}$.

A prática profissional na atenção domiciliar em um contexto de violência traz dificuldades para os profissionais que nem sempre estão preparados para lidar com tantas situações de violência contra pessoas indefesas e não encontram respaldo nos diversos serviços como polícia, judiciário, assistência social e outros, tendo em vista a necessidade de intervenções intersetoriais. Além disso, muitas vezes, a violência evidencia situações familiares já existentes que se agravam com a dependência das pessoas assistidas. Os artigos analisados abordam, principalmente violência contra os idosos, embora se reconheça a magnitude da violência contra crianças e mulheres na sociedade, mas que nem sempre estão em atenção domiciliar.

\section{Conclusão}

Dentre as situações de violência contra pacientes na $\mathrm{AD}$ mais abordadas nos artigos destacam-se contra os idosos a identificação de autonegligência e detecção e condutas de abusos, maus tratos e negligência, contra as crianças foram encontradas a violência doméstica, abuso, negligência infantil e maus tratos e contra as mulheres destaca-se a violência doméstica. A maior parte dos artigos abordava práticas relacionadas a situações de violência contra idosos e crianças e as situações de violência contra mulheres na $\mathrm{AD}$ foram menos frequentes. Destaca-se a importância de realizar estudos específicos na área da violência contra a mulher na $\mathrm{AD}$. A identificação da violência é o primeiro passo para se adotar estratégias que protejam pessoas vulneráveis, tendo em vista que, geralmente, há sinais de abusos e os profissionais de saúde devem estar atentos para que possam adotar medidas de proteção.

$\mathrm{O}$ vínculo dos profissionais de saúde com os pacientes e o conhecimento do contexto são importantes na decisão de agir sobre prováveis situações de violência. No entanto, por razões diversas, os profissionais mostram dificuldades para identificar a violência praticada e assim, as medidas protetivas podem não ser adotadas em sua prática na $\mathrm{AD}$.

A violência na $A D$, pelos achados, tem sido pouco abordada se comparada aos vários estudos sobre violência de grupos específicos, como um problema de saúde pública, além de ser necessários outros estudos sobre o tema.

\section{Colaboradores}

MA Maia participou da concepção do projeto, análise, interpretação dos dados e redação do artigo. MAC Silva participou da análise, interpretação dos dados e redação do artigo. ACO Paiva participou da redação do artigo. DM Silva participou da redação do artigo. M Alves participou da redação do artigo, revisão crítica relevante do conteúdo intelectual e aprovação final da versão a ser publicada. 


\section{Referências}

1. Cerqueira D, Lima RS, Bueno S, Valencia LI, Hanashiro O, Machado PHG, Lima AS. Atlas da violência. Rio de Janeiro: IPEA; 2017.

2. Brasil. Ministério da Saúde (MS). Caderno de atenção domiciliar. Brasília: MS; 2012.

3. Minayo MCS, Souza ER. É possível prevenir a violência? Reflexões a partir do campo da saúde pública. Cien Saude Colet 1999; 4(1):7-23.

4. Minayo MCS. Violência e saúde. Rio de Janeiro: Editora Fiocruz; 2006.

5. Brasil. Portaria no 825, de 25 de abril de 2016. Redefine a Atenção Domiciliar no âmbito do Sistema Único de Saúde (SUS) e atualiza as equipes habilitadas. Diário Oficial da União 2016; 26 abr.

6. Vognar L, Gibbs LM. Care of the Victim. Clin Geriatr Med 2014; 30(4):869-880.

7. Guzzo ACA, Cabral AC. Protocolo de Atenção Integral a crianças e adolescentes vítimas de violência: uma abordagem interdisciplinar na Saúde. Tribunal de justiça do Distrito federal e dos Territórios: Unicef; 2011.

8. Instituto Brasileiro de Geografia e Estatística (IBGE). Pesquisa nacional de saúde do escolar: 2015. Coordenação de População e Indicadores Sociais. Rio de Janeiro: IBGE; 2016.

9. Whittemore R, Knafl K. The integrative review: updated methodology. J Adv Nurs 2005; 52(5):546-553.

10. JBI. Reviewers' manual [Internet]. Adelaide: JBI; 2014 [acessado 2017 Jun 08]. Disponível em: https://joannabriggs.org/assets/docs/sumari/ReviewersManual-2014.pdf

11. Souza MT, Silva MD, Carvalho R. Integrative review: what is it? How to do it? Einstein 2010; 8(1):102-106.

12. Oxford Centre for Evidence-based Medicine: levels of evidence [Internet]. 2009 [acessado 2018 Jan 20]. Disponível em: http://www.cebm.net/oxford-centre-evidence-based-medicine-levelsevidence-march-2009

13. Pickeringa CEZ, Ridenoura K, Salaysaya Z, ReyesGastelumb D, Pierceb SJ. Identifying elder abuse \& neglect among family caregiving dyads: A cross sectional study of psychometric properties of the QualCare scale. Int J Nurs Stud 2017; 69:41-46.

14. Couture M, Israël S, Soulières M, Sasseville M. Implementing a Systematic Screening Procedure for Older Adult Mistreatment Within Individual Clinical Supervision: Is It Feasible? J Interpersonal Violence 2016; 34(13):2813-2833.

15. Sheehan OC, Ritchie CS, Fathi R, Garrigues SK, Saliba D, Leff B. Development of Quality Indicators to Address Abuse and Neglect in Home-Based Primary Care and Palliative Care. J Am Geriatr Soc 2016; 64(12):2577-2584.

16. Johnson YO. Home care nurses' experiences with and perceptions of elder self-neglect. Home Healthc Now 2015; 33(1):31-37.

17. Powers JS. Common Presentations of Elder Abuse in Health Care Settings. Clin Geriatr Med 2014; 30(4):729-741.

18. Sharps P, Alhusen JL, Bullock L, Bhandari S, Ghazarian S, Udo IE, Campbell J. Engaging and retaining abused women in perinatal home visitation programs. Pediatrics 2013; 132(Supl. 2):S134-S139.

19. Ayalon L. Reports of Elder Neglect by Older Adults, Their Family Caregivers, and Their Home Care Workers: A Test of Measurement Invariance. J Gerontol B Psychol Sci Soc Sci 2015; 70(3):432-442.
20. Cairns J, Vreugdenhil A. Working at the frontline in cases of elder abuse: 'It keeps me awake at night'. Australias J Ageing 2014; 33(1):59-62.

21. Friedman B, Santos EJ, Liebel DV, Russ AJ, Conwell Y. Longitudinal Prevalence and Correlates of Elder Mistreatment Among Older Adults Receiving Home Visiting Nursing. J Elder Abuse Negl 2015; 27(1):34-64.

22. Alonso-Marsden S, Dodge KA, O’Donnell KJ, Murphy RA, Sato JM, Christopoulos C. Family risk as a predictor of initial engagement and follow through in a universal nurse home visiting program to prevent child maltreatment. Child Abuse Negl 2013; 37(8):555-565.

23. Davidov DM, Jack SM, Frost SS, Coben JH. Mandatory reporting in the context of home visitation programs: intimate partner violence and childern's exposure to intimate partner violence. Violence Against Women 2012; 18(5):595-610.

24. Davidov DM, Nadorff MR, Jack SM, Coben JH. Nurse home visitors' perspectives of mandatory reporting of childern's exposure to intimate partner violence to child protection agencies. Public Health Nurs 2012; 29(5):412-423.

25. Ammerman RT, Peugh JL, Putnam FW, Van Ginkel JB. Predictors of treatment response in depressed mothers receiving in-home cognitive-behavioral therapy and concurrent home visiting. Behav Modif 2012; 36(4):462-481.

26. Segal L, Opie R, Dalziel K. Theory! The missing link in understanding the performance of neonatelinfant home visiting programs to prevent child maltreatment: a systematic review. Milbank Q 2012; 90(1):47106.

27. Policastro C, Payne B. Assessing the level of elder abuse knowledge pre- professionals possess: implications for the further development of university curriculum. J Elder Abuse Negl 2014; 26(1):12-30.

28. Ramos MLCO, Silva AL. Estudo Sobre a Violência Doméstica Contra a Criança em Unidades Básicas de Saúde do Município de São Paulo - Brasil. Saúde Soc 2011; 20(1):136-146.

29. Lima MCCS, Costa MCO, Bigras M, Santana MAO, Alves TDB, Nascimento OC, Silva MR. Atuação profissional da atenção básica de saúde face à identificação e notificação da violência infanto-juvenil. Rev Baiana Saúde Pública 2011; 35(Supl. 1):S118-S137.

30. Silva LMP, Ferriani MGC, Silva MAI. Atuação da enfermagem frente à violência sexual contra crianças e adolescentes. Rev Bras Enferm 2011; 64(5):919-924.

31. Costa MC, Lopes MJM. Elementos de integralidade nas práticas profissionais de saúde a mulheres rurais vítimas de violência. Rev Esc Enferm USP 2012; 46(5):1088-1095.

32. Oliveira CC, Fonseca RMGS. Práticas dos profissionais das equipes de saúde da família voltadas para as mulheres em situação de violência sexual. Rev Esc Enferm USP 2007; 41(4):605-612.

Artigo apresentado em 06/06/2018

Aprovado em 18/12/2018

Versão final apresentada em 20/12/2018 